From the Weill Cornell Medical College; Memorial Sloan-Kettering Cancer Center; Albert Einstein Comprehensive Cancer Center, New York, NY; University of Arkansas for Medical Sciences, Little Rock, AR; Nuffield Department of Clinical Laboratory Sciences, University of Oxford, Headington, Oxford, United Kingdom; University of California-San Francisco, San Francisco, CA; and National Cancer Institute, National Institutes of Health, Washington, DC

Submitted October 10, 2008; accepted March 23, 2009; published online ahead of print at www.jco.org on September 14, 2009

Supported by Grant No. CA-121947 from the National Cancer Institute to the AIDS Malignancy Consortium. A.H.B. is supported by the Leukaemia Research Fund, United Kingdom.

Authors' disclosures of potential conflicts of interest and author contributions are found at the end of this article.

Corresponding author: Ethel Cesarman, MD, PhD, Department of Pathology and Laboratory Medicine, Weill Cornell Medical College, 1300 York Ave, New York, NY 10065; e-mail: ecesarm@med .cornell.edu

The Appendix is included in the full-text version of this article, available online at www.jco.org. It is not included in the PDF version (via Adobe $®$ Reader $®$ )

(C) 2009 by American Society of Clinical Oncology

0732-183X/09/2730-5039/\$20.00

DOI: $10.1200 / J C O .2008 .20 .5450$

\title{
Immunophenotypic Analysis of AIDS-Related Diffuse Large B-Cell Lymphoma and Clinical Implications in Patients From AIDS Malignancies Consortium Clinical Trials 010 and 034
}

Amy Chadburn, April Chiu, Jeannette Y. Lee, Xia Chen, Elizabeth Hyjek, Alison H. Banham, Ariela Noy, Lawrence D. Kaplan, Joseph A. Sparano, Kishor Bhatia, and Ethel Cesarman

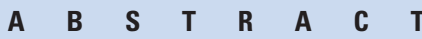

\section{Purpose}

Diffuse large B-cell lymphoma (DLBCL) represents a clinically heterogeneous disease. Models based on immunohistochemistry predict clinical outcome. These include subdivision into germinal center (GC) versus non-GC subtypes; proliferation index (measured by expression of Ki-67), and expression of BCL-2, FOXP1, or B-lymphocyte-induced maturation protein (Blimp-1)/PRDM1. We sought to determine whether immunohistochemical analyses of biopsies from patients with DLBCL having HIV infection are similarly relevant for prognosis.

\section{Patients and Methods}

We examined 81 DLBCLs from patients with AIDS in AMC010 (cyclophosphamide, doxorubicin, vincristine, and prednisone [CHOP] $\vee$ CHOP-rituximab) and AMC034 (etoposide, doxorubicin, vincristine, prednisone, and dose-adjusted cyclophosphamide plus rituximab concurrent $v$ sequential) clinical trials and compared the immunophenotype with survival data, Epstein-Barr virus (EBV) positivity, and CD4 counts.

\section{Results}

The GC and non-GC subtypes of DLBCL did not differ significantly with respect to overall survival or CD4 count at cancer presentation. EBV could be found in both subtypes of DLBCL, although less frequently in the GC subtype, and did not affect survival. Expression of FOXP1, Blimp-1/ PRDM1, or BCL-2 was not correlated with the outcome in patients with AIDS-related DLBCL.

\section{Conclusion}

These data indicate that with current treatment strategies for lymphoma and control of HIV infection, commonly used immunohistochemical markers may not be clinically relevant in HIV-infected patients with DLBCL. The only predictive immunohistochemical marker was found to be Ki-67, where a higher proliferation index was associated with better survival, suggesting a better response to therapy in patients whose tumors had higher proliferation rates.

\section{J Clin Oncol 27:5039-5048. (C) 2009 by American Society of Clinical Oncology}

\section{INTRODUCTION}

Diffuse large B-cell lymphoma (DLBCL) is the most common type of non-Hodgkin's lymphoma (NHL), accounting for 30\% to 40\% of newly diagnosed cases in the United States. ${ }^{1}$ DLBCLs are morphologically and clinically heterogeneous. On the basis of routine pathologic evaluation alone, it is often difficult to reproducibly segregate DLBCLs into clinically distinct groups. Clinical parameters, such as the International Prognostic Index, have been used to predict prognosis. ${ }^{2}$ Presumably, the International Prognostic Index reflects underlying differences in tumor biology and genetics.
Gene expression profiling has been used to stratify DLBCLs into prognostically distinct subgroups. One such schema subdivided DLBCLs into germinal center B-cell-like (GCB) DLBCLs, activated B-cell-like (ABC) DLBCLs, and heterogeneous "type 3" subtypes, 3 , which are associated with distinct genetic alterations. ${ }^{5}$ GCB-DLBCL has significantly better survival than the ABC or type 3 groups. A second model developed different expression signatures when cases were grouped according to clinical outcome, defining three subsets: oxidative phosphorylation, B-cell receptor/proliferation, and host response. ${ }^{6,7}$ Despite these gene expression advances, the expensive and technically challenging 
technology is not widely available as a routine laboratory procedure. Consequently, immunohistochemical markers that can place DLBCL into prognostically relevant categories have been identified, sometimes based on the data gleaned from the gene expression profiling research. Using tissue microarrays, CD10, BCL-6, and MUM1 have been validated as such surrogate markers to define DLBCL subtypes by their cell of origin. ${ }^{8}$ In one classification scheme, DLBCL is divided into the germinal center (GC) and non-GC groups, which have an overall survival similar to that of the GCB and $\mathrm{ABC}$ /type 3 groups identified by expression profiling, respectively. ${ }^{8}$ More recently, similar immunohistochemical algorithms have been proposed that also predict clinical behavior. ${ }^{9,10}$ Most studies reporting a better outcome of GC DLBCL have been done in patients treated with conventionally dosed chemotherapy alone (usually cyclophosphamide, doxorubicin, vincristine, and prednisone $[\mathrm{CHOP}]$ ). A better outcome was also found for GC DLBCL in poor-risk patients treated with high-dose sequential therapy and autologous stem-cell transplantation as firstline therapy. ${ }^{11}$

In patients treated with rituximab (Rituxan, Genentech, South San Francisco, CA), the clinical significance of these DLBCL subclassifications is less clear and controversial. One study showed that the prognostic difference in outcome between patients with GC or non-GC phenotypes no longer exists in patients with de novo DLBCL treated with combination $\mathrm{CHOP}$ and rituximab. ${ }^{12}$ In contrast, another study found that in patients treated with doseadjusted etoposide, doxorubicin, vincristine, prednisone, and cyclophosphamide (EPOCH) and rituximab, the GC subtype of DLBCL was associated with a better progression-free survival. ${ }^{13}$ Overall, these studies indicate that the prognostic significance of biologic markers is treatment specific.

Other specific proteins evaluated by immunohistochemistry have been shown to have equivocal prognostic validity. High proliferation rate, as determined by Ki-67 (MIB1) expression, has been found to be a strong independent predictor of poor clinical outcome in patients with DLBCL. ${ }^{14-16}$ However, other studies have reported that a low proliferative activity is associated with a shorter survival and resistance to chemotherapy in NHL. ${ }^{17,18}$ Expression of the antiapoptotic molecule BCL-2 has also been associated with a poor clinical outcome, ${ }^{16,19,20}$ although treatment with rituximab seems to eliminate the poor risk conferred by BCL-2 expression. ${ }^{13,21}$ One previous study reported that high BCL-2 expression or proliferation index does not impart a poor outcome in patients with AIDS-related DLBCL treated with dose-adjusted EPOCH. ${ }^{22}$ High-level expression of FOXP1, a transcription factor differentially expressed in resting and activated B cells, is correlated with the non-GC phenotype and has been reported to be an independent adverse prognostic marker for DLBCL. ${ }^{23-25}$ Recently, smaller FOXP1 isoforms were found in some DLBCLs; these shorter forms are induced by B-cell activation and are potentially oncogenic. ${ }^{26}$ Another protein that has received significant attention for its role in plasma cell differentiation is B-lymphocyteinduced maturation protein (Blimp-1)/PRDM1 (reviewed in ${ }^{27,28}$ ). Some DLBCLs express Blimp-1 and display more aggressive behavior, with a shorter failure-free survival. ${ }^{29}$

NHL is the second most common malignancy in HIV-infected individuals and is an AIDS-defining condition. The relative risk of NHL in people with AIDS has been estimated to be more than 100fold higher than that of the general population. ${ }^{30}$ DLBCL is the most common form of HIV-associated NHL. Although extensive investiga- tive work has been conducted on DLBCL in immunocompetent patients as reviewed above, little is known about the impact of subclassification of DLBCL in the setting of AIDS. The immunophenotypic profile and subclassification of AIDS-related DLBCL into B-cell differentiation categories has been reported in two studies that did not include clinical information. ${ }^{31,32}$ A study that included clinical data found that the non-GC phenotype was associated with a worse outcome in 89 nonuniformly treated HIV-positive patients with DLBCL. ${ }^{33}$ Only one previous study reported immunohistochemical characterization and correlation with clinical data in a panel of 25 HIV-positive patients with DLBCL who were uniformly treated with dose-adjusted EPOCH. ${ }^{22}$ To expand on that study and further evaluate whether immunophenotypic subclassification could help prognosticate cases of AIDS-related DLBCL in a larger cohort of patients, we examined cases of DLBCL from the AIDS Malignancy Consortium (AMC) clinical trials 010 (CHOP $v$ CHOP-rituximab) and AMC034 (EPOCH-rituximab concurrent $v$ sequential). We investigated whether a GC versus non-GC immunophenotype; the presence or absence of FOXP1, Blimp-1, or BCL-2 protein expression; EpsteinBarr virus (EBV) infection; or the proliferation index was correlated with overall or disease-free survival in AIDS patients with DLBCL.

\section{PATIENTS AND MATERIALS}

Eighty-one cases of HIV-associated DLBCL from AMC clinical trials 010 $(\mathrm{n}=45)$ and $034(\mathrm{n}=36)$ were included in this study. The patients in AMC010 received standard-dose CHOP, either alone or with rituximab. ${ }^{34}$ Those in AMC034 were uniformly treated with standard-dose EPOCH with either concurrent or sequential rituximab. ${ }^{35}$ This immunophenotypic study was approved by the institutional review board of Weill Cornell Medical College, and the clinical trials were approved by the review boards of all the participating institutions.

Other methods and materials are provided in the Appendix (online only).

\section{RESULTS}

\section{Patient Characteristics}

Pathology specimens from patients entered onto two clinical trials conducted by the AMC, AMC010 and 034, and classified as DLBCL by histomorphology and immunohistochemistry were included in this study. Results from these clinical trials have been reported separately. ${ }^{34,35}$ Because not all the patients from the clinical trials were included in this study, either because of a diagnosis other than DLBCL or unavailability of tissue, we first compared the characteristics of patients in our sample to those not selected for further evaluation in this study (Appendix Table A1, online only). No significant differences were found between the included and excluded cohorts.

\section{Subclassification of AIDS-Related DLBCL Into GC and Non-GC Subtypes Does Not Predict Clinical Outcome}

A total of 81 DLBCL cases were identified, but complete immunophenotypic analysis was not possible in all of these because of limited clinical material availability or failure of some antibodies. A diagram is provided in Figure 1 indicating how many cases from each 


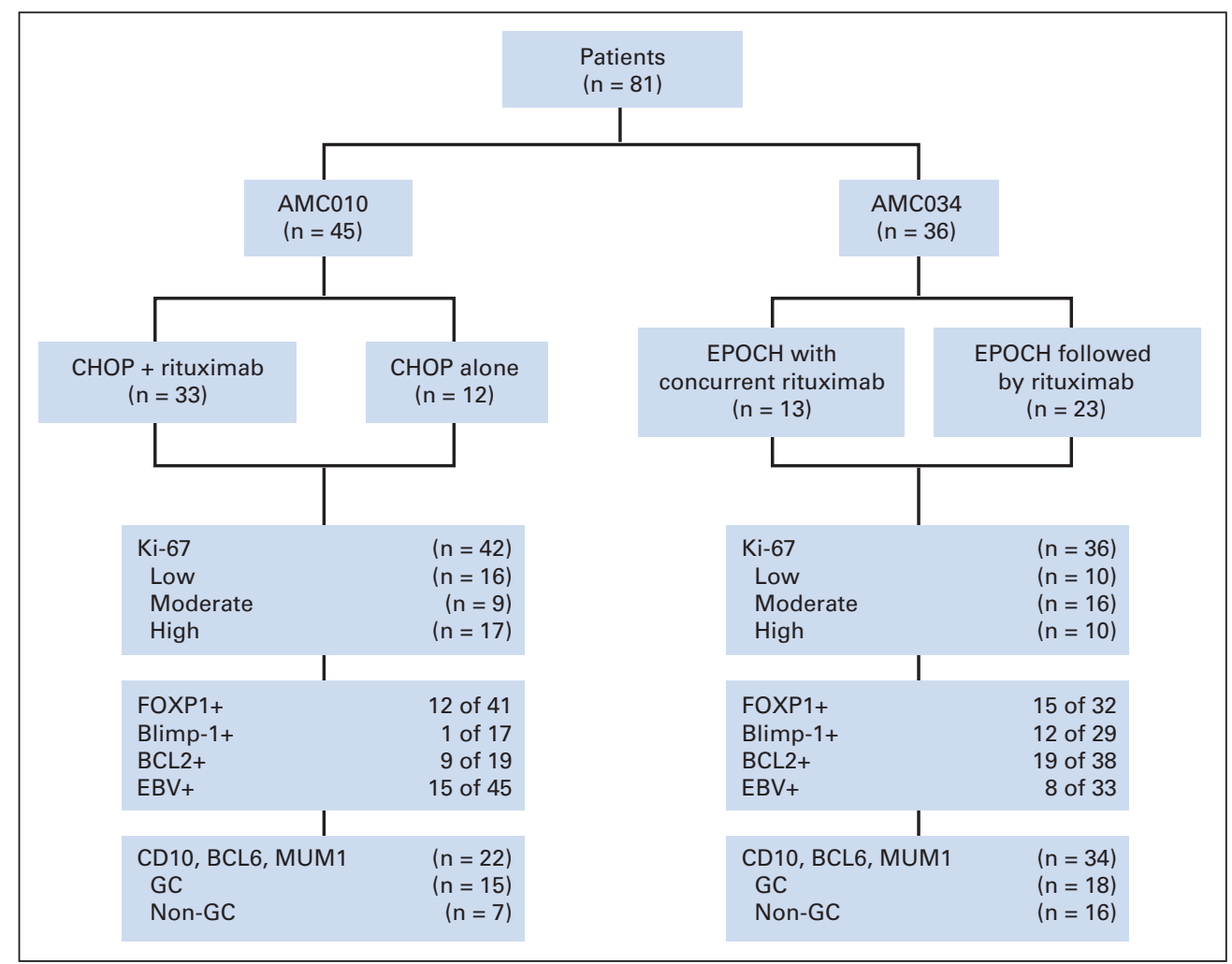

Fig 1. Number of patient samples used for statistical analysis in each subgroup. Given that not all the analyses were possible in all the specimens because of insufficient material or failure of some antibodies as a result of tissue fixation or preservation artifacts, the exact number for each analysis in each treatment subgroup varied. The number of samples for each variable and results according to clinical trial is provided. For Ki-67 staining, cases were separated into those with low proliferation index $1 \leq 60 \%$ tumor cells positive), moderate proliferation index (65\% to $85 \%$ tumor cells positive), and high proliferation index ( $\geq 95 \%$ of tumor cells positive). AMC, AIDS Malignancy Consortium; CHOP, cyclophosphamide doxorubicin, vincristine, and prednisone: $\mathrm{EPOCH}$, etoposide, doxorubicin, vincristine, prednisone, and dose-adjusted cyclophosphamide; GC, germinal center.

cohort of patients were included in each of the analyses as recommended under the proposed Reporting Recommendations for Tumor Marker Prognostic Studies criteria. ${ }^{36}$ A summary of the total cases evaluated for each independent marker and percent positivity is provided in Appendix Table A2 (online only).

GC or non-GC subtype assignment is illustrated in Figure 2A, and a representative example of each subtype is shown in Figures $2 \mathrm{~B}$ and $2 \mathrm{C}$. In 25 of 81 evaluated cases, the subtype could not be determined. Thirty-three cases (59\%) were subclassified as GC DLBCL and 23 cases $(41 \%)$ were classified as non-GC DLBCL. This contrasts with the inverse proportion reported in the HIV-negative setting using this classification: $42 \%$ for GC DLBCL and 58\% for non-GC DLBCL. ${ }^{37}$

In cases where the expression of all three antigens, CD10, BCL-6, and MUM-1, was successfully evaluated, we compared the distribution of the various combinations with the reported data from a cohort of DLBCL in immunocompetent individuals. ${ }^{8}$ In our cohort, we found more frequent coexpression of all three antigens (19\% v6\%), less frequent lack of expression of all three antigens (6\% $v 19 \%)$, and less frequent expression of BCL- 6 without expression of CD10 or MUM-1 (2\% v 14\%; Appendix Table A3, online only).

Cumulative and event-free survival was evaluated with respect to subclassification into the two main differentiation subtypes. Eventfree survival was defined as time to progression of lymphoma or death and is synonymous with progression-free survival. Overall survival is time to death regardless of cause. There was no difference in the clinical outcome between GC and non-GC DLBCL (Figs 2D and 2E).

As an alternative approach, cases were subclassified according to Amen et al, ${ }^{9}$ where either CD10 or BCL- 6 was used to define a case as GC DLBCL. Using these criteria, 44 cases were GC DLBCLs and 16 cases were considered non-GC. Among the former group, 18 were
BCL2 negative (potentially favorable). No difference in overall survival was found between GC and non-GC cases using this classification $(P=.859)$. One-year survival rate for $\mathrm{GC}$ was $70 \%(95 \% \mathrm{CI}$, $55.4 \%$ to $84.6 \%$ ) and for non-GC was $75 \%$ (95\% CI, 53.3\% to $96.7 \%$ ).

We determined whether a particular subtype of NHL is more frequent in patients with more severe immunodeficiency. Patients were divided into cohorts with less than $100 \mathrm{CD} 4$ cells/ $\mu \mathrm{L}$ (15 patients) or more than 100 cells/ $\mu \mathrm{L}$ (35 patients) and correlated with the lymphoma subtype (GC $v$ non-GC). No significant associations were found among these subgroups $(P=.11)$.

\section{Expression of FOXP1, Blimp-1, or BCL-2 Does Not Affect Clinical Outcome in AIDS-Related DLBCL}

High level of FOXP1, a transcription factor whose expression is induced in activated $\mathrm{B}$ cells, has been reported to predict a poor clinical outcome in immunocompetent patients with DLBCL. We evaluated FOXP1 expression in our cohort of patients with AIDSrelated DLBCL. Representative cases and tonsil controls are shown in Figure 3. There were no statistically significant differences in cumulative or event-free survival with respect to FOXP1 expression $(P=.79$ and $P=.57$, respectively). Furthermore, expression of FOXP1 was not correlated with the GC or non-GC subtypes of DLBCL.

Like FOXP1, Blimp-1/PRDM1 has been implicated in prognostication of DLBCL. ${ }^{29}$ Blimp-1 is a transcriptional repressor and a key regulator of terminal differentiation in B lymphocytes that is critical for plasma cell differentiation. Blimp-1 is expressed in postgerminal center B cells. Figure 4 shows representative immunohistochemistry in control tonsils and two cases of DLBCL. There was no significant difference in cumulative or event-free survival (Fig 4) with respect to Blimp-1 expression ( $P=.17$ and $P=.98$, respectively). Blimp-1 


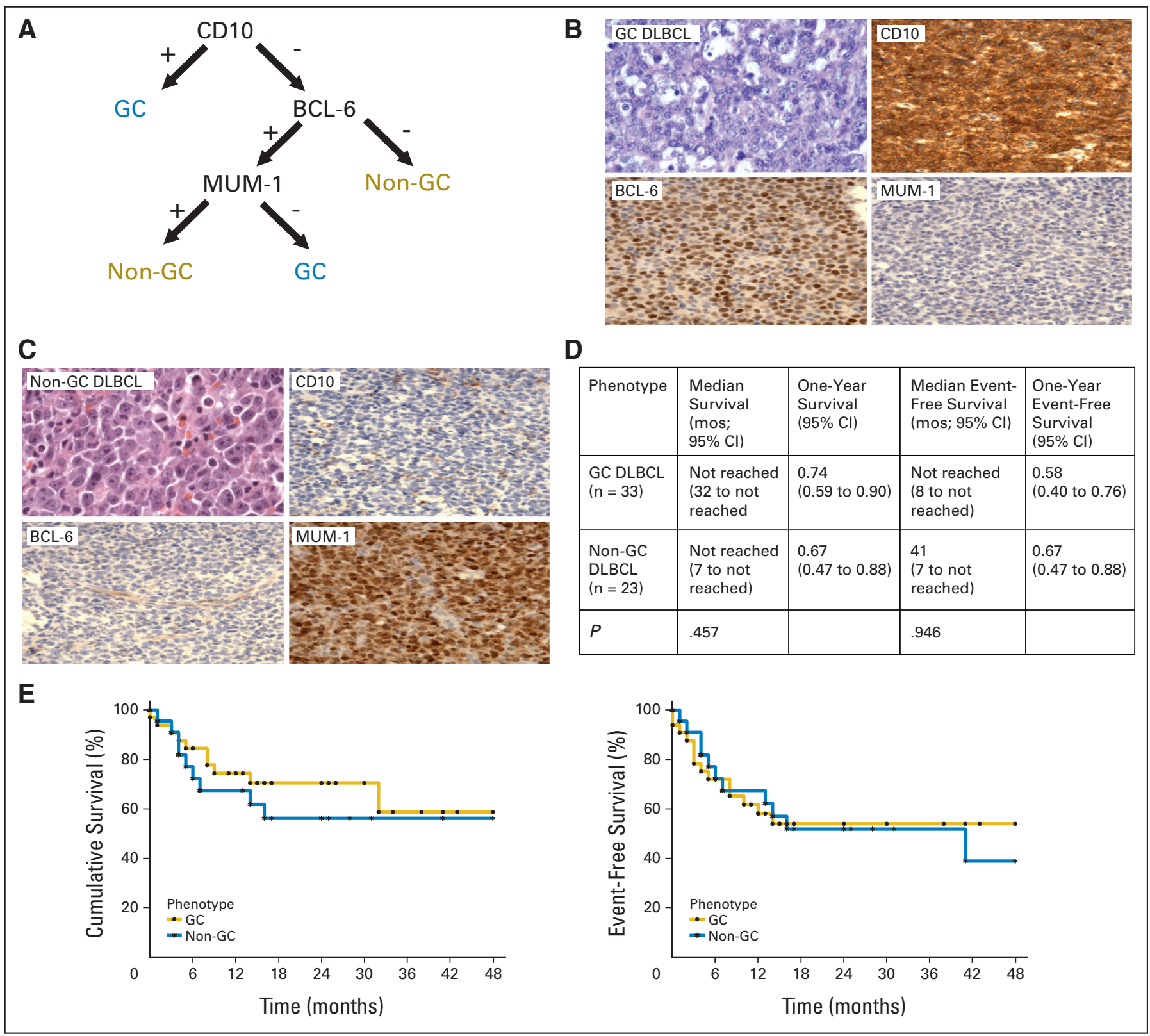

Fig 2. Subclassification of diffuse large B-cell lymphoma ( $D L B C L$ ) into differentiation subtypes and survival according to this subclassification. (A) Cases were subclassified according to the algorithm reported by Hans et al, ${ }^{8}$ as shown. (B) Histology (hematoxylin and eosin) and immunophenotype of representative case with a germinal center (GC) phenotype: CD10+, BCL-6 positive, MUM-1 negative. (C) Histology and immunophenotype of representative case with a non-GC phenotype: CD10 negative, BCL-6 negative, MUM-1 positive. The original magnification for hematoxylin and eosin was $\times 132$ and for the immunohistochemistry pictures $\times 66$. (D) Median and 1-year overall and event-free survival data. (E) Kaplan-Meier plots showing cumulative and event-free survival at 4 years of follow-up.

expression was not correlated with subtype (GC $v$ non-GC) or with FOXP1 expression.

BCL-2 is an antiapoptotic molecule that has been found to be predictive of a poor clinical outcome in non-AIDS DLBCL, ${ }^{16,19,20}$ although treatment with rituximab seems to eliminate the poor risk conferred by BCL-2 expression. ${ }^{13,21}$ We found that in our cohort, BCL-2 expression was not correlated with overall or event-free survival (not shown). In one study, ${ }^{9}$ a favorable subcategory within the GC subgroup was identified in HIV-negative patients with DLBCL when phenotyping was negative for both cyclin D1 and BCL2. Although we did not evaluate cyclin D1 expression, we determined whether the GC DLBCL cases negative for BCL2 have a favorable outcome in HIV-infected individuals. There was no significant difference in overall survival between BCL2-negative GC and other cases $(P=.51)$, with a 1 -year survival rate of $78 \%(95 \% \mathrm{CI}, 58 \%$ to $97 \%)$ and $69 \%$ (95\% CI, $54 \%$ to $84 \%)$, respectively.

\section{EBV Is Less Common in GC DLBCL But Does Not Predict Outcome}

EBV is known to be present in a significant subset of AIDSrelated lymphomas, and we found it in $29 \%$ of cases in our cohort (Appendix Table A2). Previous studies have found that this virus is 


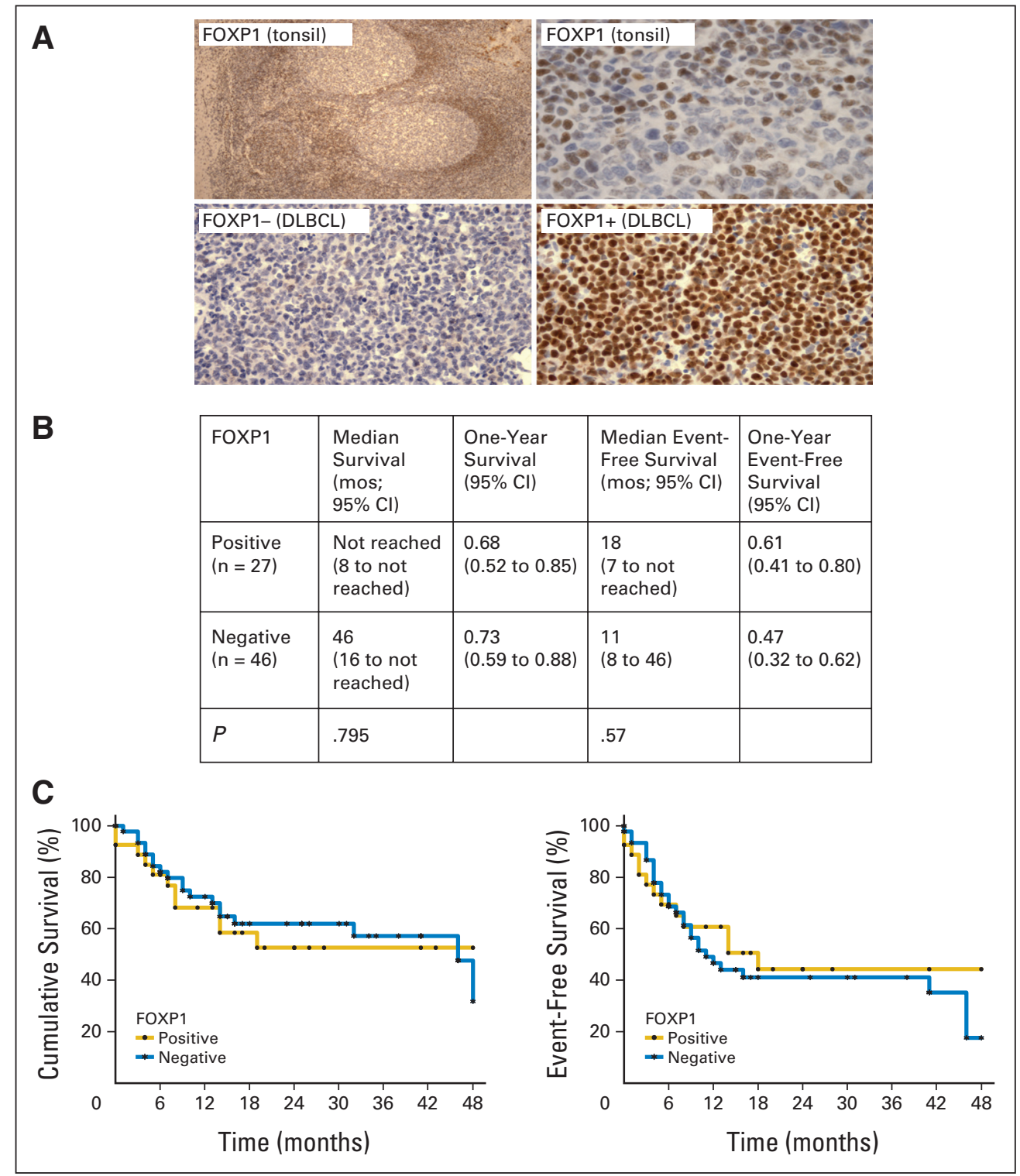

Fig 3. Immunohistochemistry for FOXP1 and impact of expression on overall and event-free survival. (A) Immunohistochemistry of FOXP1 in a tonsil (control) and two cases of AIDS-related diffuse large B-cell lymphoma (DLBCL; original magnification: tonsil, upper left, $\times 13.2$; tonsil, upper right, $\times 132$; DLBCL, lower left, $\times 66$; DLBCL, lower right, $\times 66$ ). The case on the left was considered negative, and the one on the right was scored as positive. (B) Median and 1 -year overall and event-free survival. (C) Kaplan-Meier plots of cumulative (left) and event-free survival (right)

more commonly present in cases with immunoblastic morphology that are of postgerminal center cell origin ${ }^{38}$ and could possibly impart a worse prognosis. We evaluated whether EBV is specifically associated with a specific subtype of DLBCL and whether its presence affects clinical outcome. EBV was less common in the GC DLBCLs (Fig $5 \mathrm{~A})$, although it was present in both subtypes. Classification of the AIDS-related DLBCL cases as described by Amen et $\mathrm{al}^{9}$ showed a better and statistically significant correlation with EBV status than by using the classification scheme described by Hans et al. ${ }^{8}$ The presence of EBV did not affect the cumulative or event-free survival (Fig 5B). Correlations between the presence of EBV and other immunohistochemical markers, including FOXP1 and Blimp-1, were evaluated, but no significant differences were found. In addition, contrary to the expectation that EBV would be more frequently present (and therefore more likely to play a pathogenic role) in lymphomas occurring in the most immunodeficient individuals, the patients' CD4 counts $(<100$ and $>100$ cells $/ \mu \mathrm{L})$ were not correlated with the presence or absence of EBV in their NHL specimen $(P=.47)$.

\section{High Proliferation Index Predicts a Better Clinical Outcome in AIDS-Related DLBCL}

We determined the proliferation index of the AIDS-related DLBCL cases by immunohistochemistry with Ki-67, an antigen expressed during all phases of the cell cycle, but absent from resting cells. We stratified the cases intro three different categories: high proliferation (Ki-67 > 90\% of tumor cells positive), intermediate (Ki$67=65 \%$ to $85 \%$ of tumor cells positive), and low (Ki- $67<60 \%$ of tumor cells positive). Previously, this stratification in one cohort of non-AIDS DLBCL showed that the intermediate category had a better clinical outcome. ${ }^{18} \mathrm{We}$ found a significant difference between the three groups with respect to overall survival $(P=.05)$; interestingly, a higher proliferation index was associated with improved survival (Fig 6A). A similar trend was seen for event-free survival, but this did not reach statistical significance. As the impact of proliferation index may differ among various forms of chemotherapy, and continuous infusion chemotherapy used in AMC034 may target dividing cells more effectively, we evaluated the impact of proliferation index in the two 


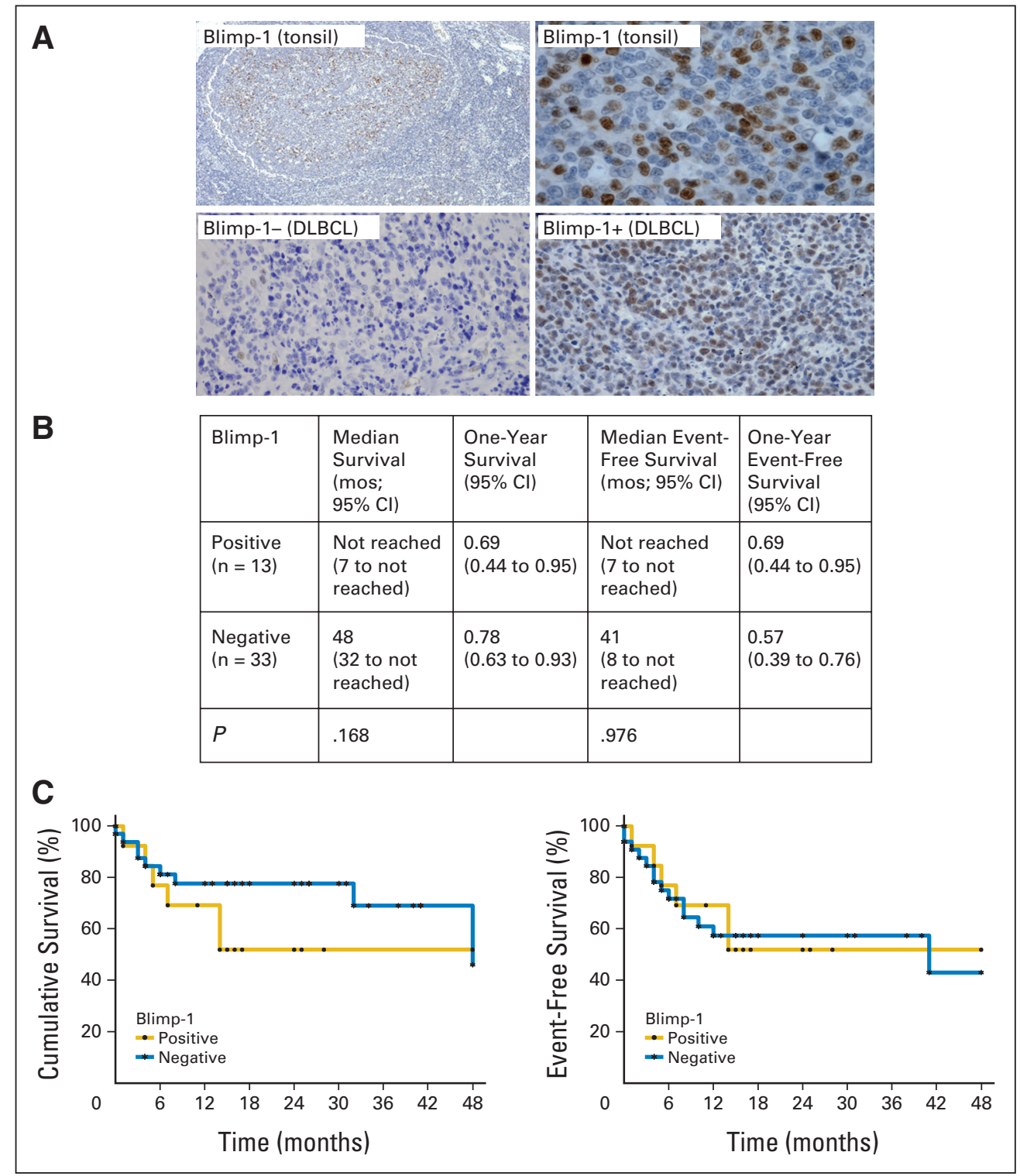

Fig 4. Immunohistochemistry for Blimp-1 and impact of expression on overall survival. (A) Immunohistochemistry of Blimp-1 in a tonsil (control) and two cases of AIDSrelated diffuse large B-cell lymphoma (DLBCL; original magnification: tonsil, upper left, $\times 6.6$; tonsil, upper right, $\times 132$; DLBCL, lower left, $\times 66$; DLBCL, lower right, $\times 66$ ). The case on the left was considered negative, and the one on the right was scored as positive. (B) Median and 1-year overall survival. (C) Kaplan-Meier plots of cumulative (left) and event-free survival (right).

separate trials (Fig 6B). A higher proliferation rate significantly predicted improved survival only in AMC034. Event-free survival in AMC034 showed a similar trend, but did not reach statistical significance (not shown).

\section{DISCUSSION}

The introduction of highly active antiretroviral therapy (HAART) has resulted in a decline in the incidence of HIV-associated lymphoma, but the risk remains increased. ${ }^{39,40}$ Current thought is that HIVinfected patients should be treated as aggressively as immunocompetent patients with the same lymphoma type, but the prognosis remains worse in patients with AIDS, as infectious complications are more likely to occur, particularly in those with lower CD4 counts. ${ }^{41}$

In this study, subclassification of AIDS-associated DLBCLs into GC or non-GC type using immunohistochemical methods ${ }^{8,9}$ did not predict outcome. Although some patients in AMC010 did not receive rituximab, the number was too small for assessment of whether patients with non-GC DLBCL in this treatment arm had an adverse outcome. The expression of antigens found to define a poor risk category in non-AIDS patients, including FOXP1, BCL-2, and Blimp-1, did not prognosticate survival in this cohort of patients with AIDS-related DLBCL. This finding is similar to that of Little et al, ${ }^{22}$ who found that BCL-2 and p53 overexpression failed to affect survival of patients with AIDS-related lymphoma treated with dose-adjusted EPOCH. For the classification of AIDS-related lymphomas, we recommend the use of CD20, CD3, CD10, BCL-2, BCL-6, and MUM-1, Ki-67, EBV-EBER, and KSHV-LANA, which usually allow the separation of DLBCL, Burkitt's lymphoma, T-cell lymphoma, and extracavitary primary effusion lymphoma. This panel includes CD10, BCL-6, and MUM-1, which would also allow for further subclassification into GC and non-GC subtypes, but our current observations suggest that this further subtyping may not supply any clinically useful information in the setting of the current therapeutic modalities. 


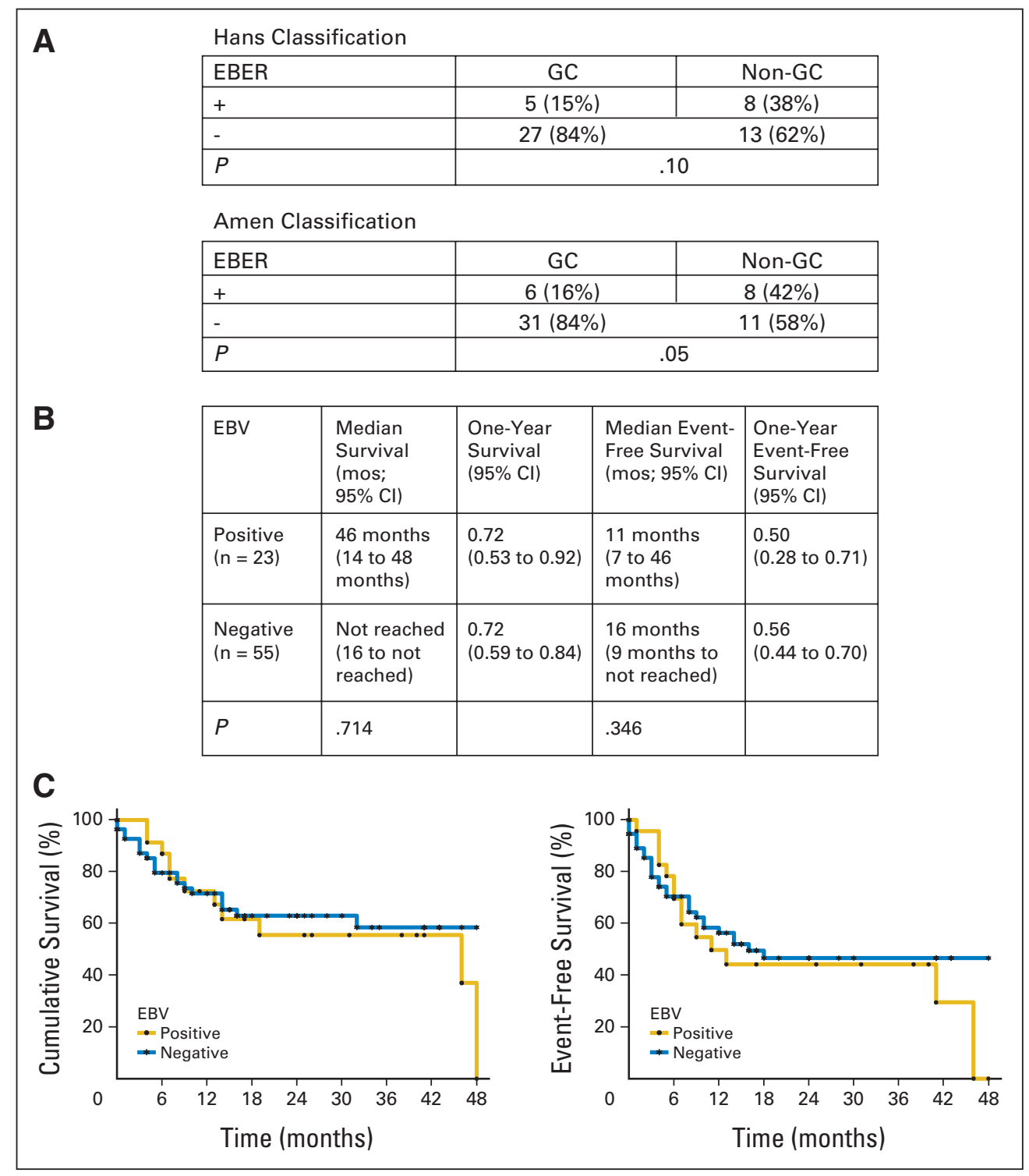

Fig 5. Epstein-Barr virus (EBV) and diffuse large B-cell lymphoma (DLBCL) subtype and survival. (A) The presence of EBV in germinal center (GC) and non-GC subtypes according to two different classifications strategies ${ }^{8,9}$ was evaluated. In both schemes, EBV positivity was more common in non-GC DLBCL, but this trend only reached statistical significance when cases were classified according to Amen et al. $^{9}$ (B) Overall survival according to EBV status was evaluated; median and 1 -year survival are shown. (C) KaplanMeier plots of cumulative (left) and eventfree survival (right).
In this cohort of HIV-positive patients, the relative proportion of GC DLBCLs was higher than in immunocompetent patients. A previous study also reported more cases expressing the germinal center cell antigens CD10 and BCL- 6 in a panel 25 AIDS-related DLBCLs, as compared with a similar cohort in HIV-negative patients. ${ }^{22}$ A recent study evaluating 12 AIDS-related and 27 non-AIDS-related DLBCLs showed that AIDS-related DLBCLs exhibit an immunophenotype intermediate between the GC and activated B-cell types of DLBCL present in immunocompetent patients, concluding that the AIDSrelated DLBCLs might have a unique pathophysiology. ${ }^{32}$ Our data confirm a slightly different distribution of antigen expression, with more frequent coexpression of both GC cell antigens (CD10 and BCL-6) and a post-GC cell marker (MUM-1).

The presence of EBV in our cohort was approximately 30\%, consistent with the published ranges for centroblastic DLBCL ( $20 \%$ to $40 \%$ ), although in the immunoblastic category, EBV is reported to be more frequently present. The vast majority of our cases had centroblastic morphology. Nine cases had immunoblastic histology, and among these, five were positive for EBV. The incidence of primary CNS lymphomas has markedly decreased since the onset of HAART. ${ }^{42}$ It has been postulated that improved immune surveillance of EBV viral proteins that are both oncogenic and immunogenic prevents these tumors from growing. A Japanese study showed that EBV-positive lymphomas decreased from $88 \%$ in the pre-HAART era to $58 \%$ in the HAART era, but did not differ significantly between HAART users (73\%) and nonusers (74\%). ${ }^{43}$ Contrary to our predictions, we show that the frequency of EBV in DLBCLs is not increased in patients who are more severely immunocompromised. One preHAART study indicated that EBV was slightly more common in patients with lower CD4 counts. ${ }^{44}$ It is possible that more subtle immunologic abnormalities than CD4 counts allow the EBV-infected lymphoma cells to proliferate. ${ }^{45,46}$

We found that higher proliferation rates, as determined by expression of $\mathrm{Ki}-67$, are associated with a better clinical outcome. This is 


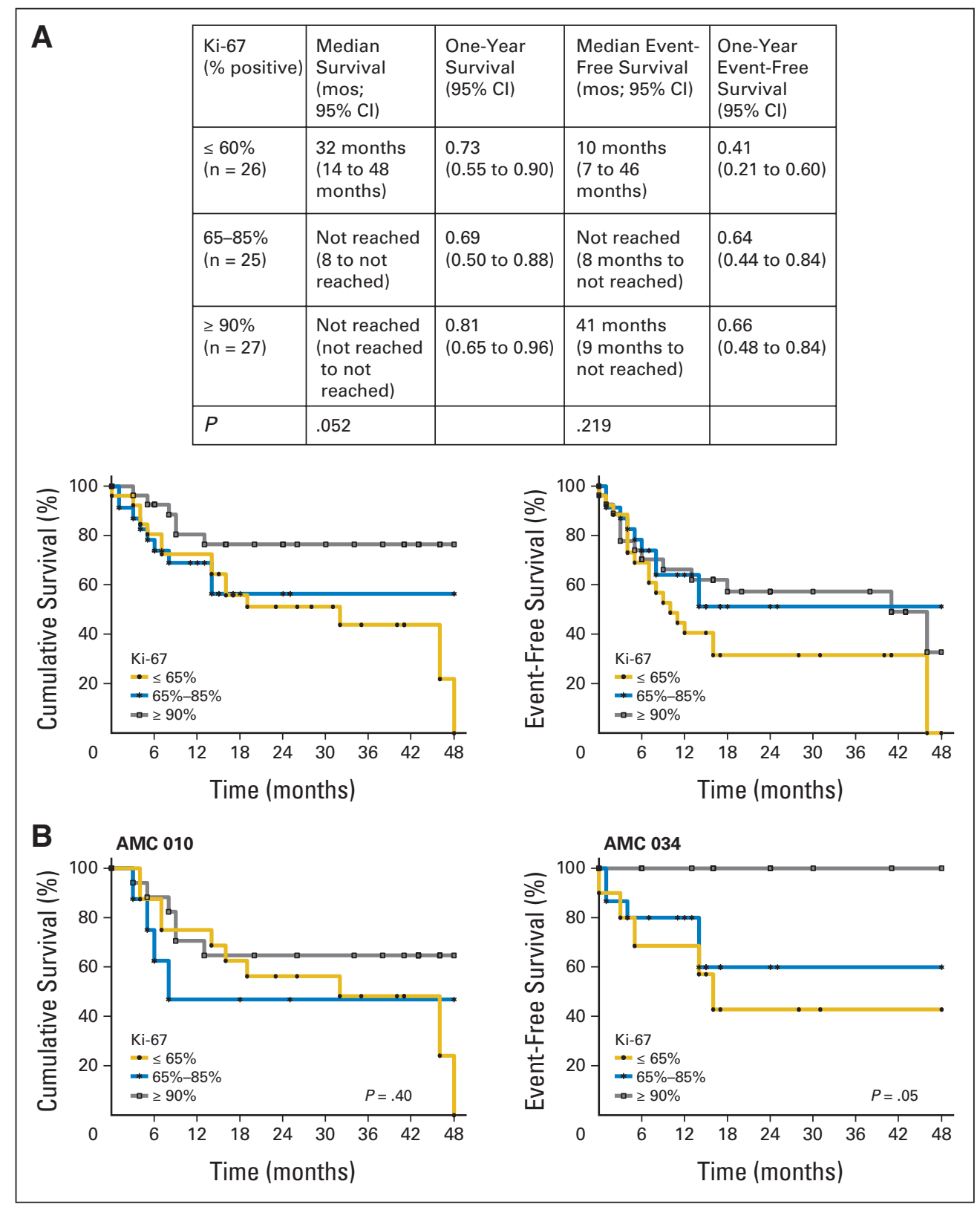

Fig 6. Proliferation index and survival. Proliferation was assessed by staining with antibodies to $\mathrm{Ki}-67$ and divided into three subgroups according to the percentage of positivity in the tumor cells. (A) The median and 1 -year overall and event-free survival for all patients evaluated are shown in the upper panel, and corresponding Kaplan-Meier plots are shown in the lower panel. (B) Analysis was performed separately on patients from AIDS Malignancy Consortium (AMC) 010 and AMC034; Kaplan-Meier plots of cumulative (left) and event-free survival (right). The log-rank $P$ value for these analyses is indicated within the graphs.

probably related to a better response to continuous infusional combination chemotherapy, which targets proliferating cells. EPOCH given over 5 days may eliminate all the cells dividing during this time period and therefore is more likely to be effective in tumors with a rapid cellular turnover.

This immunophenotypic study of a large cohort of HIVinfected patients with DLBCL revealed significant differences from similar studies on immunocompetent individuals with DLBCL. We found a lack of predictive impact of most immunohistochemical markers, whereas a higher proliferation rate imparted a better prognosis. These finding have significant implications for pathologic diagnosis in terms of the immunohistochemical panels used during diagnostic classification. Our findings also have clinical relevance, as different chemotherapeutic modalities or scheduling regimens may be more effective based on the proliferation index of the lymphoma cells.

\section{AUTHORS' DISCLOSURES OF POTENTIAL CONFLICTS OF INTEREST}

Although all authors completed the disclosure declaration, the following author(s) indicated a financial or other interest that is relevant to the subject matter under consideration in this article. Certain relationships marked with a " $U$ " are those for which no compensation was received; those relationships marked with a " $C$ " were compensated. For a detailed description of the disclosure categories, or for more information about ASCO's conflict of interest policy, please refer to the Author Disclosure Declaration and the Disclosures of Potential Conflicts of Interest section in Information for Contributors. 
Employment or Leadership Position: None Consultant or Advisory Role: None Stock Ownership: None Honoraria: None Research Funding: None Expert Testimony: None Other Remuneration: Alison H. Banham, Financial interest in the JC12 antibody (to FOXP1) as a consequence of commercial licencing

\section{AUTHOR CONTRIBUTIONS}

Conception and design: Amy Chadburn, Joseph A. Sparano, Kishor Bhatia, Ethel Cesarman Financial support: Ethel Cesarman
Provision of study materials or patients: Alison H. Banham, Ariela Noy, Lawrence D. Kaplan, Joseph A. Sparano

Collection and assembly of data: Amy Chadburn, April Chiu, Jeannette Y. Lee, Xia Chen, Elizabeth Hyjek, Joseph A. Sparano, Ethel Cesarman Data analysis and interpretation: Amy Chadburn, April Chiu, Jeannette Y. Lee, Alison H. Banham, Elizabeth Hyjek, Ariela Noy, Joseph A. Sparano, Kishor Bhatia, Ethel Cesarman

Manuscript writing: April Chiu, Alison H. Banham, Ariela Noy, Ethel Cesarman

Final approval of manuscript: Amy Chadburn, April Chiu, Jeannette Y. Lee, Xia Chen, Alison H. Banham, Elizabeth Hyjek, Ariela Noy, Lawrence D. Kaplan, Joseph A. Sparano, Kishor Bhatia, Ethel Cesarman

\section{REFERENCES}

1. A clinical evaluation of the International Lymphoma Study Group classification of non-Hodgkin's lymphoma: The Non-Hodgkin's Lymphoma Classification Project. Blood 89:3909-3918, 1997

2. A predictive model for aggressive nonHodgkin's lymphoma: The International NonHodgkin's Lymphoma Prognostic Factors Project. N Engl J Med 329:987-994, 1993

3. Alizadeh $A A$, Eisen $M B$, Davis $R E$, et al: Distinct types of diffuse large B-cell lymphoma identified by gene expression profiling. Nature 403: 503-511, 2000

4. Rosenwald A, Wright G, Chan WC, et al: The use of molecular profiling to predict survival after chemotherapy for diffuse large-B-cell lymphoma. N Engl J Med 346:1937-1947, 2002

5. Lenz G, Wright GW, Emre NC, et al: Molecular subtypes of diffuse large B-cell lymphoma arise by distinct genetic pathways. Proc Natl Acad Sci U S A 105:13520-13525, 2008

6. Shipp MA, Ross KN, Tamayo P, et al: Diffuse large B-cell lymphoma outcome prediction by geneexpression profiling and supervised machine learning. Nat Med 8:68-74, 2002

7. Monti S, Savage KJ, Kutok JL, et al: Molecular profiling of diffuse large B-cell lymphoma identifies robust subtypes including one characterized by host inflammatory response. Blood 105:1851-1861, 2005

8. Hans CP, Weisenburger DD, Vose JM, et al: A significant diffuse component predicts for inferior survival in grade 3 follicular lymphoma, but cytologic subtypes do not predict survival. Blood 101:23632367, 2003

9. Amen $F$, Horncastle D, Elderfield $K$, et al: Absence of cyclin-D2 and Bcl-2 expression within the germinal centre type of diffuse large B-cell lymphoma identifies a very good prognostic subgroup of patients. Histopathology 51:70-79, 2007

10. Chang CC, McClintock S, Cleveland RP, et al: Immunohistochemical expression patterns of germinal center and activation B-cell markers correlate with prognosis in diffuse large B-cell lymphoma. Am J Surg Pathol 28:464-470, 2004

11. van Imhoff GW, Boerma EJ, van der Holt B, et al: Prognostic impact of germinal center-associated proteins and chromosomal breakpoints in poor-risk diffuse large B-cell lymphoma. J Clin Oncol 24:41354142,2006

12. Nyman $H$, Adde $M$, Karjalainen-Lindsberg $M L$, et al: Prognostic impact of immunohistochemically defined germinal center phenotype in diffuse large B-cell lymphoma patients treated with immunochemotherapy. Blood 109:4930-4935, 2007

13. Wilson $W H$, Dunleavy $K$, Pittaluga $S$, et al: Phase II study of dose-adjusted EPOCH and ritux- imab in untreated diffuse large B-Cell lymphoma with analysis of germinal center and post-germinal center biomarkers. J Clin Oncol 26:2717-2724, 2008

14. Grogan TM, Lippman SM, Spier CM, et al: Independent prognostic significance of a nuclear proliferation antigen in diffuse large cell lymphomas as determined by the monoclonal antibody Ki-67. Blood 71:1157-1160, 1988

15. Miller TP, Grogan TM, Dahlberg $S$, et al: Prognostic significance of the Ki-67-associated proliferative antigen in aggressive non-Hodgkin's lymphomas: A prospective Southwest Oncology Group trial. Blood 83:1460-1466, 1994

16. Sanchez E, Chacon I, Plaza MM, et al: Clinical outcome in diffuse large B-cell lymphoma is dependent on the relationship between different cell-cycle regulator proteins. J Clin Oncol 16:1931-1939, 1998

17. Wilson $W H$, Teruya-Feldstein J, Fest $T$, et al: Relationship of p53, bcl-2, and tumor proliferation to clinical drug resistance in non-Hodgkin's lymphomas. Blood 89:601-609, 1997

18. Jerkeman $M$, Anderson $H$, Dictor $M$, et al: Assessment of biological prognostic factors provides clinically relevant information in patients with diffuse large B-cell lymphoma: A Nordic Lymphoma Group study. Ann Hematol 83:414-419, 2004

19. Sjö LD, Poulsen CB, Hansen M, et al: Profiling of diffuse large B-cell lymphoma by immunohistochemistry: Identification of prognostic subgroups. Eur J Haematol 79:501-507, 2007

20. Veelken H, Vik Dannheim S, Schulte Moenting $J$, et al: Immunophenotype as prognostic factor for diffuse large B-cell lymphoma in patients undergoing clinical risk-adapted therapy. Ann Oncol 18:931-939, 2007

21. Shivakumar $L$, Armitage JO: $\mathrm{Bcl}-2$ gene expression as a predictor of outcome in diffuse large B-cell lymphoma. Clin Lymphoma Myeloma 6:455457, 2006

22. Little RF, Pittaluga $S$, Grant $N$, et al: Highly effective treatment of acquired immunodeficiency syndrome-related lymphoma with dose-adjusted EPOCH: Impact of antiretroviral therapy suspension and tumor biology. Blood 101:4653-4659, 2003

23. Banham AH, Connors JM, Brown PJ, et al: Expression of the FOXP1 transcription factor is strongly associated with inferior survival in patients with diffuse large B-cell lymphoma. Clin Cancer Res 11:1065-1072, 2005

24. Barrans SL, Fenton JA, Banham A, et al: Strong expression of FOXP1 identifies a distinct subset of diffuse large B-cell lymphoma (DLBCL) patients with poor outcome. Blood 104:2933-2935, 2004

25. Shaffer AL, Rosenwald A, Staudt LM: Lymphoid malignancies: The dark side of B-cell differentiation. Nat Rev Immunol 2:920-932, 2002
26. Brown PJ, Ashe SL, Leich E, et al: Potentially oncogenic B-cell activation-induced smaller isoforms of FOXP1 are highly expressed in the activated B cell-like subtype of DLBCL. Blood 111:2816-2824, 2008

27. Martins G, Calame K: Regulation and functions of Blimp-1 in T and B lymphocytes. Annu Rev Immunol 26:133-169, 2008

28. Nutt SL, Fairfax KA, Kallies A: BLIMP1 guides the fate of effector $B$ and T cells. Nat Rev Immunol 7:923-927, 2007

29. Garcia JF, Roncador G, Garcia JF, et al: PRDM1/BLIMP-1 expression in multiple B and T-cell Iymphoma. Haematologica 91:467-474, 2006

30. Goedert JJ: The epidemiology of acquired immunodeficiency syndrome malignancies. Semin Oncol 27:390-401, 2000

31. Carbone A, Gloghini A, Larocca LM, et al: Expression profile of MUM1/IRF4, BCL-6, and CD138/syndecan-1 defines novel histogenetic subsets of human immunodeficiency virus-related lymphomas. Blood 97:744-751, 2001

32. Madan R, Gormley R, Dulau A, et al: AIDS and non-AIDS diffuse large B-cell lymphomas express different antigen profiles. Mod Pathol 19:438-446, 2006

33. Hoffmann $C$, Tiemann $M$, Schrader $C$, et al: AIDS-related B-cell lymphoma (ARL): Correlation of prognosis with differentiation profiles assessed by immunophenotyping. Blood 106:1762-1769, 2005

34. Kaplan LD, Lee JY, Ambinder RF, et al: Rituximab does not improve clinical outcome in a randomized phase 3 trial of $\mathrm{CHOP}$ with or without rituximab in patients with HIV-associated nonHodgkin lymphoma: AIDS-Malignancies Consortium Trial 010. Blood 106:1538-1543, 2005

35. Levine AM, JL, Kaplan L, et al: Efficacy and toxicity of concurrent rituximab plus infusional $\mathrm{EPOCH}$ in HIV-associated lymphoma: AIDS Malignancy Consortium Trial 034. J Clin Oncol 26:460s, 2008 (suppl; abstr 8527)

36. McShane LM, Altman DG, Sauerbrei W, et al: Reporting recommendations for tumor marker prognostic studies. J Clin Oncol 23:9067-9072, 2005

37. Hans CP, Weisenburger DD, Greiner TC, et al: Confirmation of the molecular classification of diffuse large B-cell lymphoma by immunohistochemistry using a tissue microarray. Blood 103:275-282, 2004

38. Carbone A: Emerging pathways in the development of AIDS-related lymphomas. Lancet Oncol 4:22-29, 2003

39. Biggar RJ, Chaturvedi AK, Goedert JJ, et al: AIDS-related cancer and severity of immunosuppression in persons with AIDS. J Natl Cancer Inst 99:962-972, 2007 
40. Engels EA, Biggar RJ, Hall HI, et al: Cancer risk in people infected with human immunodeficiency virus in the United States. Int $\mathrm{J}$ Cancer 123:187-194, 2008

41. Sparano JA: HIV-associated lymphoma: The evidence for treating aggressively but with caution. Curr Opin Oncol 19:458-463, 2007

42. Gates AE, Kaplan LD: AIDS malignancies in the era of highly active antiretroviral therapy. Oncology (Williston Park) 16:441-451, 456, 459, 2002
43. Hishima T, Oyaizu N, Fujii T, et al: Decrease in Epstein-Barr virus-positive AIDS-related lymphoma in the era of highly active antiretroviral therapy. Microbes Infect 8:1301-1307, 2006

44. Kersten MJ, Van Gorp J, Pals ST, et al: Expression of Epstein-Barr virus latent genes and adhesion molecules in AIDS-related nonHodgkin's lymphomas: Correlation with histology and CD4-cell number. Leuk Lymphoma 30:515524,1998
45. Pallesen G, Hamilton-Dutoit SJ, Rowe M, et al: Expression of Epstein-Barr virus latent gene products in tumour cells of Hodgkin's disease. Lancet 337:320-322, 1991

46. Rea D, Delecluse HJ, Hamilton-Dutoit SJ, et al: Epstein-Barr virus latent and replicative gene expression in post-transplant lymphoproliferative disorders and AIDS-related non-Hodgkin's lymphomas: French Study Group of Pathology for HIV-associated Tumors. Ann Oncol 5:113-116, 1994 (suppl 1) 